\title{
Comparison of survival time of Hawley and Vacuum-formed retainers in orthodontic patients- a randomized clinical trial
}

Seyed Hossein Moslemzadeh ${ }^{1}$, Aydin Sohrabi ${ }^{2}$, Ali Rafighi ${ }^{3}$, Morteza Ghojazadeh ${ }^{4}$, Somaieh Rahmanian*5

1. Assistant Professor, Department of Orthodontics, School of Dentistry, Tabriz University of Medical Sciences, Tabriz, Iran.hmoslemzade@yahoo.com

2. Associate Professor, Department of orthodontics, School of dentistry, Tabriz University of medical science. ay_sohrabi@yahoo.com

3. Assistant Professor, Department of orthodontics, School of faculty of dentistry, Tabriz University of medical science, rafighi_ali@yahoo.com

4. Assistant Professor of Physiology (MPH, PhD), Tabriz University of Medical Sciences, Tabriz, Iran. ghojazadehm@hotmail.com

5. Assistant Professor, Department of Orthodontics, School of Dentistry, Kurdistan University of Medical Sciences, Kurdistan, Iran. somaieh.rahmanian93@gmail.com

\begin{abstract}
Background: Maintaining the results of orthodontic treatment and keeping the teeth in the corrected position is a great challenge in orthodontics. This study aimed to compare the survival time of three types of retainers including Hawley, 1-mm Vacuum-Formed (VF), and 1.5$\mathrm{mm}$ VF within 6-month period. Methods: In this randomized clinical study, 152 patients were allocated into three groups to receive one type of the retainers. They were visited 1, 3, and 6 months after retainer delivery and checked for breakage, loss, local perforation, and discoloration from the patient's and clinician's point of view as indicators of failure. Chi-square and Fisher's exact tests were used as appropriated. Result: The results revealed that breakage was among the main reasons of failure of retainers within 6 months, which was statistically significantly different between Hawley and VF retainers, as well as between 1-mm and 1.5-mm VF retainers in the three intervals $(p<0.05)$. Concerning the loss of retainer and discoloration from the clinician's point of view, there was no significant difference between the retainers in any interval $(p>0.05)$. Assessing the discoloration from the patient's point of view revealed statistically significant differences between Hawley and VF retainers within the first month; however, the difference was not significant at the third and sixth months $(p<0.05)$. The difference between the two thicknesses of VF retainer was not significant in any interval $(p>0.05)$. By the end of the sixth month, some of the VF retainers had perforation; while, perforation was not observed in Hawley retainers.

Conclusion: Considering the higher breakage rate of 1-mm VF, 1.5-mm VF seems the retainer if choice.
\end{abstract}

Key words: survival time; retainer; Hawley retainer; Vacuum-formed retainer;

\section{Corresponding author:}

Somaieh Rahmanian

Assistant Professor, Department of Orthodontics, School of Dentistry, Kurdistan University of Medical Sciences, Kurdistan, Iran.somaieh.rahmanian93@gmail.com

Receive date: 2016-11-01 | Accept date: 2016-12-21 | Publish date: 2017-02-10

DOI: 10.7575/aiac.abcmed.17.05.01.02 


\section{Introduction}

The success of treatment in orthodontics is determined by facial esthetics, occlusion, and stability.(1) Maintaining the results of orthodontic treatment and keeping the teeth in the corrected position is a great challenge in orthodintics. As stated by Angle, retention problems are greater than the difficulties being encountered in orthodontic treatments.(2)

Several attempts to find the best strategy of stabilizing the teeth in the new position after orthodontic treatment and to prevent relapse have resulted in introduction of various fixed and removable retainers.(3) Among the removable retainers, Hawley, Vacuum-Formed, Begg, and Positioner are more frequently used than others.

Choosing the appropriate type of retainer for each patient requires considering several factors, namely, the patient's pre-treatment conditions, age, oral health, and periodontal status, as well as his preference.(4) Since there are not adequate scientific evidence, the decision depends mostly on the clinician's personal preferences. To improve the treatment stability and patient cooperation, the orthodontists mainly adopt a combined use of various removable and fixed retainers.(5)

Having been inroduced nearly hundred years ago, Hawley is among the most prevalent appliances of removable retention in orthodontic clinics.(6) The patients might find this retainer esthetically inconvenient because the labial bow that is made up of round steel wire contacts the labial surface of 4 or 6 anterior teeth.(1)

Attempting to overcome the esthetic problem of Hawley retainers, Sheridane and colleagues introduced Essix appliances or Vacuum-Formed (VF) retainers, in 1993, as a replacement for the conventional retainers. These transparent retainers have become increasingly popular in recent years due to several reasons such as ease of fabrication, improved esthetic, and lower cost. $(7,8)$ The patients mostly prefer the nearly-invisible VF retainers. Despite multiple advantages, these transparent retainers often become opaque, eroded, cracked, or ruptured over time. $(5,7,9)$

Several studies have investigated different aspects of success and failure of Hawley and VF removable retainers. Having compared the efficiency of these two, some researchers declared that the retention created by Hawley is insufficient due to the single-point contact of the wire on the labial surface and an acrylic mass in proximity of lingual cervix of anterior teeth. Meanwhile, VF retainer thoroughly encapsulates the dentition and the superior part of alveolus, thus providing better retention.(7)

Several studies have also assessed and compared the role of these two types of retainer in maintaining the intercanine width, intermolar width, rotation, overjet, overbite, $(10)$ and occlusal contacts. $(11,12)$ Some investigations compared their speech articulation during retention.(13)

Most orthodontists prescribe a period of full-time wear of retainers and recommend continuing their part-time use throughout the life; hence, endurance and persistence of retainer are of paramount importance. If shortlived, the economic burden increases on the patient, consequently the patient cooperation and treatment outcome would be negatively impressed.(14)

Multiple studies has been carried out on the endurance of bonded retainers; whereas, only few prospective investigations assessed the survival time of removable retainers. Previous systematic studies mentioned the lack of adequate clinical evidence about the survival time of removable retainers. Comprehensive 
clinical studies have been suggested to evaluate and compare the survival rate of Hawley and VF removable retainers. $(15,16)$

Nor was found any inclusive clinical study on the effect of different thicknesses of VF retainers on their survival rate. Hence, the present clinical study was designed to assess and compare the survival rate of Hawley, and the most commonly-used thicknesses of $\mathrm{VF}$ removable retainers (1-mm and $1.5-\mathrm{mm} \mathrm{VF}$ ) within 6 months.

\section{Materials and Method}

In this multicentric clinical study, the target population was patients whose comprehensive orthodontic treatment was performed and finished in the researchers' private office. The patients were gradually enrolled with informed consent until the required number of subjects was achieved.

According to Hichens et al. (2007), the prevalence of fracture of Hawley retainer during the retention period was $19 \%$. Considering a clinically significant difference of $15 \%, \alpha=0.05$, and power $=80 \%$, the required number of samples should be 105 . With regard to thesample attrition in long-term studies, a total of 152 patients were recruited to increase the reliability of the study.

The inclusion criteria were having finished the orthodontic treatment in an optimal occlusion, indication of using removable retainer, and the patient's willingness to participate in the study. The exclusion criteria were having several missing teeth and the need for prosthetic replacement, bruxism, syndromic diseases (e.g. cleft lip and cleft palate, hemifacial microsomia), allergic sensitivity to acrylic resin, temporomandibular joint problems, oral habits that would cause malocclusion, patients with maxillary expansion treatments, preterm debonding, and generalized spacing.
The subjects were randomly allocated into three groups according to the table of random numbers retrieved from www.randomizer.org website for each center. The patients in each of the three groups received fixed bonded retainer in the mandible. In the maxilla, the first group was administered with Hawley removable retainer, the second group received 1.5-mm VF retainer, and the third group received 1-mm VF retainer.

By the end of orthodontic treatment when the subjects were ready for debonding, the brackets were removed, impression was taken by using alginate material (Tropicalgin Zhermark; Italy), and retainers were fabricated on the plaster model. The removable Hawley retainers prepared in each center consisted of Adams Clasp on the first molar teeth, labial bow with 28-mil SS wire (Dentaurum, Germany) and self-cure acrylic (Dentaurum; Germany). The VF retainers made of 1 - and $1.5-\mathrm{mm}$ thermoplastic platens (3A Co.; Korea) were trimmed in a way that they lie along the gingival margin in the buccal surface, $3-4 \mathrm{~mm}$ beyond the gingival margin in the lingual surface, and cover the most distal tooth in the occlusal surface.

A time interval of 1-2-days was considered between taking the impressions and placing the retainers. Prior to being delivered, the retainer was checked for accordance, presence of any plausible damage, and patient comfort. The patients were trained to wear the retainer at all times, except when eating. Regardless of the type of retainer, the patients in each center received identical practicalinstructions on how and when to use and health care.Theprinted instruction was also handed out to the patients

In order to determine the survival time of retainers, special forms were prepared to record breakage (wire components or base of the retainer), lose, perforation or any type of abrasion that would result in perforation observable with naked eyes. Minor cracks on 
the surfaces were not considered as breakage unless their spreading would make the retainer useless.

The patients were followed up at 1, 3, and 6 months after placing the appliances to evaluate the survival time of retainers. Since the assessment was performed by clinicians, clinician blindness was impossible. It should be noted that the survival time was only calculated for the main retainer (the first retainer).

The obtained data were statistically analyzed by using SPSS software, version 22. First, the descriptive parameters were calculated in each group. Then to evaluate the frequency of breakage in the three types of retainer at different time intervals, Chi-square and Fisher's exact tests were used $(\alpha=0.05)$.

\section{Results}

Out of 152 participants, 117 were females and 35 were males. There were 52 subjects in Hawley group (39 females and 13 males), 52 in 1-mm VF group (40 females, 12 males), and 48in 1.5-mm VF group (38 females, 10 males). The patients were sex-matched, there was no statistically significant difference between the two sexes regarding the distribution of retainers $(p=0.885)$. Matching was also done on the retainers distributed among the clinicians and no statistically significant difference was noted ( $p=0.397)$. Having not referred at the scheduled appointment, a number of patients were excluded from the study.

Breakage

The most common reason of replacing the retainer within the 6-month period was breakage of the retainer. Table 1 shows that 1 month after retainer delivery, $4.5 \%$ breakage was observed in 1.5-mm VF retainer, and 20\% in 1-mm VF group. No retainer breakagewas noted in Hawley group. Based on the results of Fisher's exact test, breakage was significantly different between Hawley and VF groups 1, 3, and 6 months after retainer delivery $(p=0.009$, $0=0.042$, and $p=0.017$, respectively). However, significantly different breakages were observed between different thicknesses of VF retainers only after 1 month ( $p=0.031)$; their difference was not significant at the third and sixth months $(p=0.227$ and $p=0.717$, respectively).The highest rate of breakage in 6month period was noted in 1-mm VF retainer (41.2\%)

\begin{tabular}{|c|c|c|c|c|c|c|c|c|}
\hline \multirow{4}{*}{ 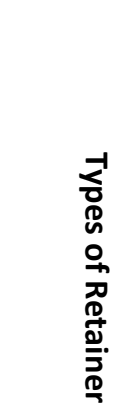 } & \multirow{2}{*}{\multicolumn{2}{|c|}{ Breakage }} & \multicolumn{2}{|c|}{1 month } & \multicolumn{2}{|c|}{3 months } & \multicolumn{2}{|c|}{6 months } \\
\hline & & & & No & Yes & No & Yes & No \\
\hline & Hawley & $\mathrm{N}(\%)$ & $0(0.0)$ & $46(100)$ & $1(3.2)$ & $30(96.8)$ & $1(5.6)$ & $17(94.4)$ \\
\hline & VFR $1.5 \mathrm{~mm}$ & $\mathrm{~N}(\%)$ & $2(4.5)$ & $42(92.5)$ & $4(11.1)$ & $32(88.9)$ & $4(33.3)$ & $8(66.7)$ \\
\hline & VFR1mm & $\mathrm{N}(\%)$ & $10(20)$ & $40(80)$ & $9(23.1)$ & 30 (76.9) & $7(41.2)$ & $10(58.8)$ \\
\hline \multicolumn{3}{|c|}{$\begin{array}{l}{ }^{*} p \text { value to compare Hawley and } \\
\text { VF }\end{array}$} & \multicolumn{2}{|c|}{0.009} & \multicolumn{2}{|c|}{0.042} & \multicolumn{2}{|c|}{0.017} \\
\hline \multicolumn{3}{|c|}{${ }^{*} p$ value to compare types of VF } & \multicolumn{2}{|c|}{0.031} & \multicolumn{2}{|c|}{0.227} & \multicolumn{2}{|c|}{0.717} \\
\hline
\end{tabular}

*p value from Fisher's exact test

Table 1: Frequency of breakage of different retainers in the three intervals 


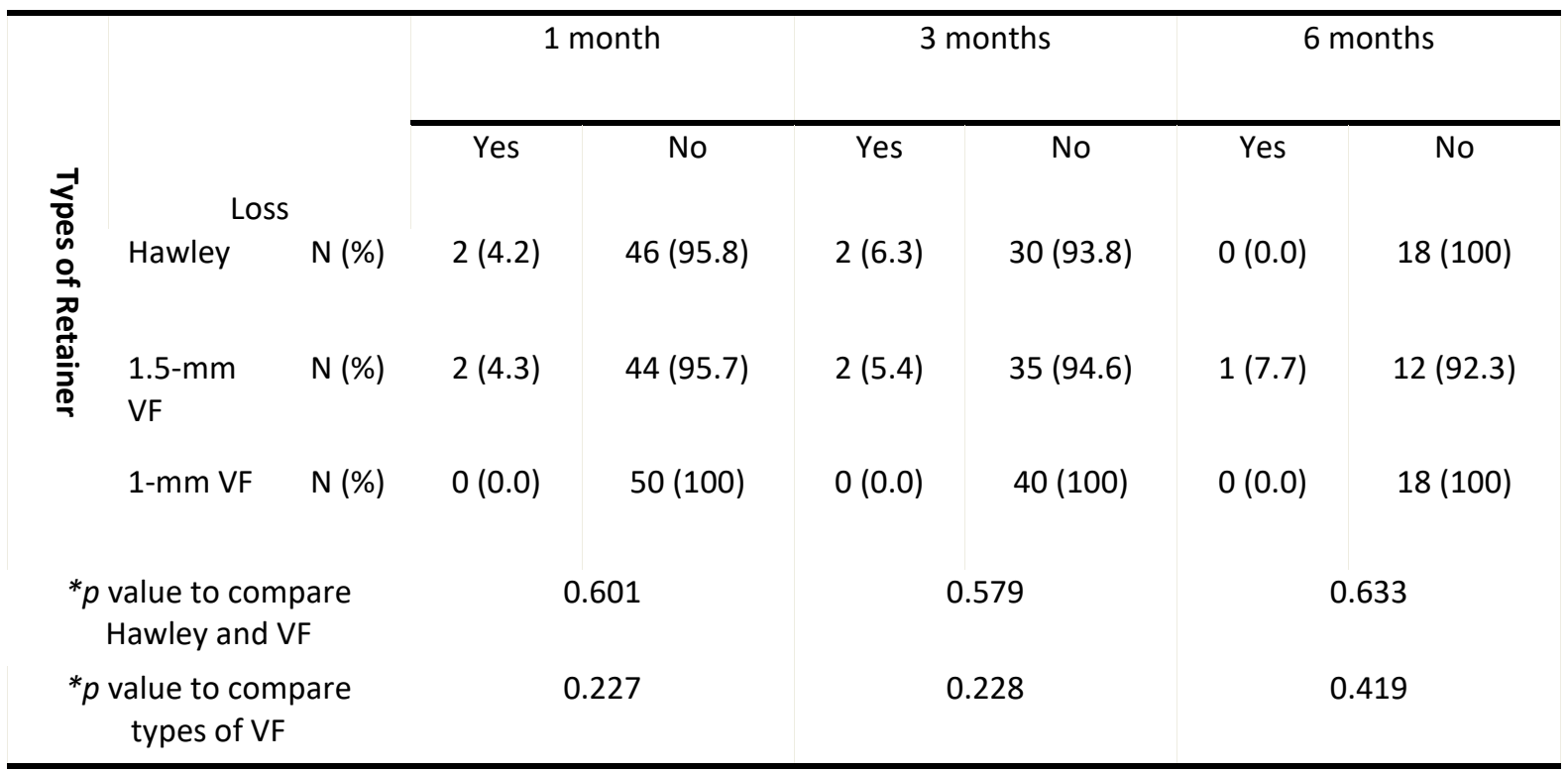

*p value from Fisher's exact test

Table 2: Distribution of lost retainers within the three time intervals

\section{Loss}

Table 2 shows that losing the retainer within the first month was $4.2 \%$ in Hawley, $4.3 \%$ in 1.5-mm VF, and zero in 1-mm VF group. No significant difference was noted between loss of the Hawley and VFretainers at 1-, 3-, and 6month intervals $(p=0.601, p=0.579$, and $p=0.633$, respectively). No significant difference was noted between 1-mm and 1.5-mm VF retainers at the three intervals $(p=0.227$, $p=0.228$, and $p=0.419$, respectively).

\section{Discoloration}

Being considered as another influential factor of the retainer survival time, discoloration was investigated from both the patient's and clinician's the point of view in the current study.

\section{Discoloration from the patients' point of view}

The obtained results demonstrated that discoloration within the first month was $2.2 \%$ in Hawley, $18.2 \%$ in $1.5-\mathrm{mm} \mathrm{VF}$, and $22.4 \%$ in $1-$ $\mathrm{mm} V \mathrm{~F}$ group. Accordingly, discoloration was significantly different between Hawley and VF retainersin the first month $(p=0.004)$; and insignificant at the third and sixth months $(p=$ $106, p=0.140$, respectively). As demonstrated in Table 3, there was no significant difference between discoloration of1-mm and 1.5-mm VF retainers in the first, third, and sixth months ( $p=0.797, p=0.793$, and $p=459$, respectively).

\section{Discoloration from the clinician's point of view}

From the clinicians' point of view after one month, discoloration was $10.9 \%$ in Hawley, $18.2 \%$ in $1.5-\mathrm{mm} \mathrm{VF}$, and $18.4 \%$ in $1-\mathrm{mm} \mathrm{VF}$ group. No significant difference existed between Hawley and VF retainers in terms of discoloration in the first, third, and sixth month ( $p=0.328, p=0.501$, and $p=0.769$, respectively). Nor was significant difference noted between 1-mm and 1.5-mm VF retainers at any of the time intervals $(p=1.000, p=0.627$, and $p=$ 0.252 , respectively) (Table 4 ).

\section{Perforation}

Assessing the frequency of perforations created in retainers revealed no significant difference between Hawley and VF retainersin the first month after retainer delivery $(P=1.000)$. In the third month, the frequency of 
perforations was $17.1 \%$ in $1.5-\mathrm{mm} \mathrm{VF}, 30.6 \%$ in 1-mm VF, and $0 \%$ in Hawley retainers (no perforation), indicating a statistically significant difference between Hawley and VF retainers at this interval $(p=0.02)$. This difference was also significant in the sixth month $(p=0.04)$. Comparison of the frequency of perforation revealed no significant difference between 1$\mathrm{mm}$ and $1.5-\mathrm{mm} \mathrm{VF}$ in any of the time intervals $(p=0.496, p=0.267$, and $p=0.673$, respectively) (Table 5).

\begin{tabular}{|c|c|c|c|c|c|c|c|c|}
\hline \multirow{3}{*}{ 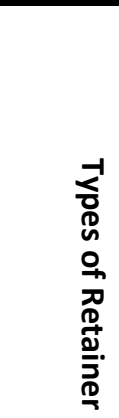 } & \multirow{2}{*}{\multicolumn{2}{|c|}{ Discoloration }} & \multicolumn{2}{|c|}{1 month } & \multicolumn{2}{|c|}{3 months } & \multicolumn{2}{|c|}{6 months } \\
\hline & & & Yes & No & Yes & No & Yes & No \\
\hline & 1.5-mm VF & $\mathrm{N}(\%)$ & $1(2.2)$ & $\begin{array}{l}45(97.8) \\
36(81.8)\end{array}$ & $\begin{array}{l}7(23.3) \\
9(25.7)\end{array}$ & $\begin{array}{l}23(76.7) \\
26(74.3)\end{array}$ & $\begin{array}{l}6(33.3) \\
8(66.7)\end{array}$ & $12(66.7)$ \\
\hline & 1-mm VF & $\mathrm{N}(\%)$ & $11(22.4)$ & $38(77.6)$ & $11(30.6)$ & $25(69.4)$ & $8(50.0)$ & $8(50.0)$ \\
\hline \multicolumn{3}{|c|}{$\begin{array}{l}{ }^{*} p \text { value to compare Hawley and } \\
\text { VF }\end{array}$} & \multicolumn{2}{|c|}{0.004} & \multicolumn{2}{|c|}{0.106} & \multicolumn{2}{|c|}{0.140} \\
\hline \multicolumn{3}{|c|}{${ }^{*} p$ value to compare types of VF } & \multicolumn{2}{|c|}{0.797} & \multicolumn{2}{|c|}{0.793} & \multicolumn{2}{|c|}{0.459} \\
\hline
\end{tabular}

Table 3: Distribution of retainer discoloration from the patients' point of view at the three time intervals

\begin{tabular}{|c|c|c|c|c|c|c|c|c|}
\hline \multirow{3}{*}{ 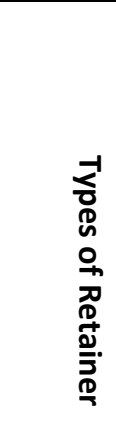 } & & & \multicolumn{2}{|c|}{1 month } & \multicolumn{2}{|c|}{3 months } & \multicolumn{2}{|c|}{6 months } \\
\hline & \multicolumn{2}{|c|}{ Discoloration } & Yes & No & Yes & No & Yes & No \\
\hline & 1.5-mm VF & N (\%) & 5 (10.9) & $\begin{array}{l}41 \text { (89.1) } \\
36(81.8)\end{array}$ & $12(34.3)$ & $\begin{array}{l}21(70.0) \\
23(65.7)\end{array}$ & $8(33.3)$ & $10(55.6)$ \\
\hline & 1-mm VF & $\mathrm{N}(\%)$ & 9 (18.4) & $40(81.6)$ & 15 (41.7) & $21(58.3)$ & $6(37.5)$ & $10(62.5)$ \\
\hline \multicolumn{3}{|c|}{${ }^{*} p$ value to compare Hawley and VF } & \multicolumn{2}{|c|}{0.328} & \multicolumn{2}{|c|}{0.501} & \multicolumn{2}{|c|}{0.769} \\
\hline \multicolumn{3}{|c|}{${ }^{*} p$ value to compare types of VF } & \multicolumn{2}{|c|}{1.000} & \multicolumn{2}{|c|}{0.627} & \multicolumn{2}{|c|}{0.252} \\
\hline
\end{tabular}

${ }^{*} p$ value from Fisher's exact test 
Table 4: Distribution of retainer discoloration from the clinician's point of view within the three time intervals

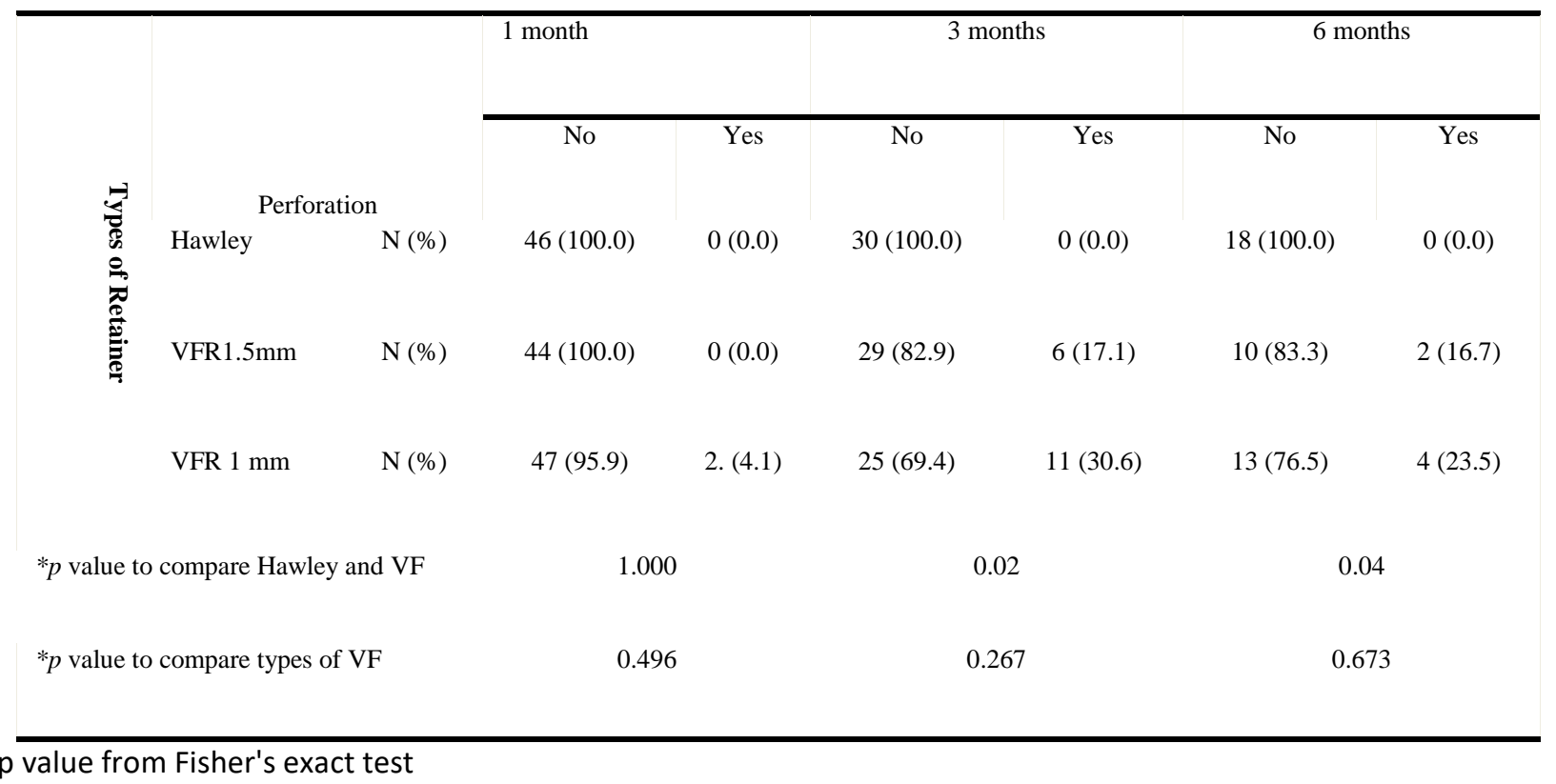

Table 5: Comparison of the frequency of perforations in the three study groups at different time intervals

Six months after retainer delivery, $16.7 \%$ of 1.5-mm VF retainers were perforated and they all had 2 perforations. On the other side, $23.5 \%$ of 1 -mm VF retainers were perforated, $25 \%$ of which had 1 perforation, and $75 \%$ had more than 3 perforations.

\section{Discussion}

The results of this study revealed that VF retainers were more susceptible to breakage than Hawley retainer in 1-, 3-, and 6-month intervals $(p=0.009, p=0.042$, and $p=0.017$, respectively). Within the first month, 1-mm VF retainers experienced more breakage than 1.5$m m$ VF retainers $(p=0.031)$. However, they had no statistically significant difference at the third and sixth months $(p=0.227$ and $p=0.717$, respectively).

With respect to the shape of Hawley retainers, as well as the relatively high coverage of palatal surface in this type of retainer, lower breakage compared with the VF retainers is expected. Comparing the two types of VF retainers in the first month, breakage was higher in 1-mm VF than that in 1.5-mm type; however, in the third and sixth months, they had no statistically significant difference. It might indicate the breakageresistance of 1.5$\mathrm{mm} V \mathrm{~F}$ in the first month, deterioration of this resistance and increased breakage in the following months.

Concerning the losing of retainer and discoloration from the clinician's point of view, there was no significant difference between VF and Hawley retainers, nor between the two thicknesses of VF retainers ( $p>0.05)$.

In terms of discoloration from the patient's point of view within the first month, VF retainer represented more discoloration than Hawley retainer $(p=0.004)$. Yet, the difference was not significant in the following months $(p=0.106$ and $p=0.140$, respectively). In the same regard, the two types of VF retainer were not significantly different in any of time intervals 
( $p=0.797, p=0.793$, and $p=0.459$, respectively). The higher discoloration of VF retainer compared with Hawleywas expected in the first month. In the following months, discoloration was still higher in VF groups; although, the difference was not statistically significant. It can be concluded that discoloration occurs earlier in VF retainer and later in Hawley.

Perforation was not observed in any of Hawley retainers; whereas, a considerable percent of VF retainers were perforated. These findings are justifiable according to the shape of Hawley retainers and the fact that it does not cover the occlusal surface of teeth.

There are only few studies on the survival time of removable retainers whose results can be compared with those of the current study. One of the prominent findings of this study was the statistically considerably different breakage between Hawley and VF retainers, and on the other hand between 1-mm and 1.5-mm VF retainers.

Sun J et al. reported no statistically considerable difference between the survival time of Hawley and 0.75-mm VF retainers.(17) That study did not separately investigated different causes and factors that affected the survival time of retainers. They reported the results under the general title of survival time. Furthermore, mandibular retainers (Hawley and VF) were also included in the study. Apparently, their results cannot be comparable to ours.

Based on our results, losing the retainers was not considerably different between Hawley and VF retainers; whereas, Sun J et al. reported the rate of lost retainer to be higher in transparent retainers than that in Hawley. This contradiction might be due to the difference in the time intervals, which was 1,3 , and 6 months in our study; while, Sun J et al. assessed this factor after one year. In other words, longtermwearof the retainer that probably decreases the daily hours of wearing the retainer, increases the possibility of losing VF retainer because of transparency and lower visibility.

Hichens et al. (2007) evaluated the breakage in Hawley and VF retainers within 6 months, and reported higher breakage rate in Hawley retainers.(18)Such difference with the current study can be attributed to the fact that they did not clearly explained the details of VF and Hawley retainers, besides their different definition of breakage.

Mc Dermott (2008) mentioned breakage $(28 \%)$, abrasion (28\%), and losing (14\%) as the main problems of using VF retainers.(19) In the present study, breakage, loss, and perforation caused by abrasion were the major causes of failure of VF retainers.

Campbell et al. (2009) reported that the main reason for replacing the VF retainers within one year was over-abrasion. Likewise, we observed that abrasion-caused perforation was among the reasons of failure of VF retainers.

In a study, Pratt reported that $10 \%$ of all participants lost their retainers within 2 years.(20) In our study, losing was evaluated as a reason of failure of the studied retainers; however, the retainers had no significant difference in terms of being lost within 6 months. Concerning other reasons for replacement of the retainer, namely perforation and discoloration of the retainer, there was no similar clinical study to be compared with the present one.

\section{Conclusion}

With respect to the results of this study, it can be concluded that:

- concerning the breakage, there were statistically significant differences between Hawley and VF retainers, and also between 1-mm and 1.5-mm VF 
retainers within the three time

intervals.

- There wasno statistically significant difference between the studied retainers within the three time intervals concerning losing the retainer and discoloration from the clinician's point of view.Nevertheless, discoloration from the patient's point of view was found to be significantly different between Hawley and VF retainers within the first month. Yet, the two thicknesses of VF retainer had no statistically significant difference in any of the studied time intervals.

- By the end of the sixth month, some of VF retainers had perforation; whereas, Hawley retainers had no perforation.

\section{References}

1.Proffit WR, Fields HW, Sarver DM. Contemporary orthodontics,5th ed. Mosby Elsevier; 2013.p.611-14.

2. Case CS, Principles of retention in orthodontia. Am J Orthod Dentofacial Orthop. 2003;124(4):352-61

3. Edman Tynelius G, Bondemark L, Lilja-Karlander E. A randomized controlled trial of three orthodontic retention methods in Class I four premolar extraction cases-stability after 2 years in retention. Orthodontics \& Craniofacial Research. 2013;16(2):105-15.

4. Bibona K, Shroff B, Best AM, Lindauer SJ. Factors affecting orthodontists' management of the retention phase. Angle Orthodontist. 2013;84(2):225-30

5. Rinchuse DJ, Sheridan J. Orthodontic retention and stability: A clinical perspective. J Clin Orthod. 2007;41(3):125-32

6. Mai W, Meng H, Jiang Y, Huang C, Li M, Yuan K, et al. Comparison of vacuum-formed and Hawley retainers: a systematic review. Am J Orthod Dentofacial Orthop. 2014;145(6):720-27

7. Demir A, Babacan H, Nalcacı R, Topcuoglu T. Comparison of retention characteristics of Essix and Hawley retainers. K JO. 2012;42(5):255-62.

8. RowlandH, Hichens L, Williams A, Hills D, Killingback N, Ewings P, et al. The effectiveness of Hawley and vacuumformed retainers: a single-center randomized controlled trial. Am J Orthod Dentofacial Orthop. 2007;132(6):730-37. 9. Pratt MC, Lindstrom AF. Patient compliance with orthodontic retainers in the postretention phase. Am J Orthod Dentofacial Orthop. 2011;140(2):196-1

10. Barlin S, Smith R, Reed R, Sandy J, Ireland AJ. A retrospective randomized double-blind comparison study of the effectiveness of Hawley vs Vacuum-formed retainers. Angle orthodontist. 2011;81(3):404-9

11. Sari Z, Uysal T, Basciftci FA, Inan O. Occlusal contact changes with removable and bonded retainers in a 1-year retention period. Angle Orthodontist. 2009;79(5):867-72.

12. Dincer M, Isik A. Effects of thermoplastic retainers on occlusal contacts. Eur J Orthod. 2010;32(1):6-10.

13. Haydar B, Karabulut G, Özkan S, Aksoy AÜ, Ciğer S. Effects of retainers on the articulation of speech. Am J orthod dentofacial orthop. 1996;110(5):535-40.

14. Sauget E, Covell Jr DA, Boero RP, Lieber WS. Comparison of occlusal contacts with use of Hawley and clear overlay retainers. Angle Orthodontist. 1997;67(3):223-30

15. Littlewood SJ, Doubleday B, Bearn DR, Worthington HV. Orthodontic retention: A systematic review. JOrthod. 2006;33(3):205-12.

16. Littlewood SJ, Doubleday B, Bearn DR, Worthington HV. Retention procedures for stabilising tooth position after treatment with orthodontic braces (Review). The Cochrane Library. 2016;1

17. Sun J, Yu Y, Liu M, Chen L, Li H, Zhang L, et al. Survival Time Comparison between Hawley and Clear Overlay Retainers A Randomized Trial. Journal of dental research. 2011;90(10):1197-201.

18. Hichens L, Rowland H, Williams A, Hollinghurst S, Ewings $P$, Clark S, et al. Cost-effectiveness and patient satisfaction: Hawley and vacuum-formed retainers. The Eur J Orthod.2007;29(4):730-37

19.McDermott P, Millett D, Field D, Van Den Heuvel A, Erfida I. Lower incisor retention with fixed or vacuum-formed retainers. J Dent Res. 2008;87

20. Pratt MC, Kluemper GT, Hartsfield JK, Fardo D, Nash DA. Evaluation of retention protocols among members of the American Association of Orthodontists in the United States. Am J Orthod Dentofacial Orthop. 2011;140(4):520-26 